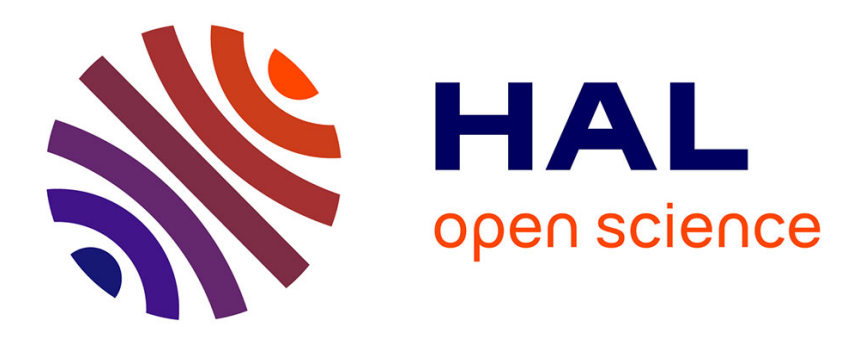

\title{
A modelling framework for finite strain magneto-viscoelasticity
}

Nedjar Boumediene

\section{To cite this version:}

Nedjar Boumediene. A modelling framework for finite strain magneto-viscoelasticity. Mathematics and Mechanics of Solids, 2019, 25p. 10.1177/1081286519873963 . hal-02303386v2

\section{HAL Id: hal-02303386 \\ https://hal.science/hal-02303386v2}

Submitted on 15 Oct 2019

HAL is a multi-disciplinary open access archive for the deposit and dissemination of scientific research documents, whether they are published or not. The documents may come from teaching and research institutions in France or abroad, or from public or private research centers.
L'archive ouverte pluridisciplinaire HAL, est destinée au dépôt et à la diffusion de documents scientifiques de niveau recherche, publiés ou non, émanant des établissements d'enseignement et de recherche français ou étrangers, des laboratoires publics ou privés. 


\title{
A modelling framework for finite strain magneto-viscoelasticity
}

\section{Boumediene Nedjar ${ }^{1}$}

\begin{abstract}
The main objective of this contribution is the theoretical modelling of magnetisable materials that exhibit viscoelastic properties. The current state of the art in the mathematical modelling of nonlinear magneto-mechanics in deformable media can be easily integrated within the unified framework of continuum thermodynamics that is crucial in setting the convenient forms for the constitutive laws and evolution equations. Due to the soft nature of the materials we have in mind, the finite strain range is a priori adopted and, in this first approach, only isotropic materials are considered. We adopt the nowadays well-accepted multiplicative split of the deformation gradient which, moreover, gives rise to an intermediate configuration. Herein, the novelty resides in the fact that the magnetic field vectors are transported onto the above mentioned intermediate configuration and, therefore, can be implicitly decomposed. The proposed formulation is based on the magnetic induction as main independent variable for the magnetic part of the problem. An alternative formulation based on the magnetic field as main independent variable can easily be deduced, but this latter will not be considered in this paper for the sake of clarity. A very simple model example that agrees with the laws of thermodynamics is proposed for the purpose of demonstration to study some phenomena qualitatively.
\end{abstract}

\section{Keywords}

Magneto-active soft materials, Large deformation, Multiplicative viscoelasticity, Continuum thermodynamics 


\section{Introduction}

Magneto-active polymers (MAPs) belong to the very important class of emerging new materials that deform under magnetic stimuli. They are mostly composites of soft polymer matrix impregnated with magnetically permeable particles, typically iron particles in micro- or nano-meter size. In general, the response to magnetic fields can be devided into two categories based on the property of the matrix part of the material: they can give large and prompt deformation, or they can change their mechanical properties with moderate straining. The two features have received considerable attention in recent years due to their potential applications including, for instance, sensors, actuators, and medicine, see for example ${ }^{1-7}$ among many others.

In parallel, the mathematical modelling of the coupling of electromagnetic fields in deformable materials has also been an area of active research, see for example ${ }^{8-16}$. Fully coupled nonlinear field theories have been developed with constitutive formulations based on augmented free energy functions. In particular, it has been shown that any one of the magnetic induction, magnetic field, or magnetization vectors can be used as independent variable, the other two being obtained through constitutive relations, see $^{17}$ for details about this topic. The relevant equations are based on the pioneering works of ${ }^{18-20}$ among others.

This work aims at developing a continuum field theory for the behaviour of magnetically sensitive materials that experience viscoelasticity, see for example ${ }^{21,22}$. Indeed, the response to magnetic stimuli can in general not be instantaneous for all MAPs as that has been established experimentally, e.g. see the recent works $i^{23,24}$. The finite strain range is a priori assumed due to the compliant nature of such materials that, in general, respond with large deformations. Herein, we adopt the multiplicative decomposition of the deformation gradient into an elastically relaxing part and a viscous part as nowadays widely used in the literature, see for example ${ }^{25-30}$. This gives rise to an intermediate configuration on which the magnetic field vectors can be transported, and implicitly decomposed. Very few models exist in the literature that couple the magnetic field and viscoelasticity. Notice that recently in ${ }^{31}$, the magnetoviscoelasticity has also been formulated for incompressible materials based on an a priori decomposition of the magnetic induction vector, and all the continuum thermodynamic developments therein follow by

\footnotetext{
${ }^{1}$ Université Paris-Est, IFSTTAR/MAST/EMGCU, France

Corresponding author:

Boumediene Nedjar, Université Paris-Est, IFSTTAR/MAST/EMGCU, 14-20 Boulevard Newton, Cité Descartes, Champs sur Marne, 77447 Marne-la-Vallée Cedex 2, France.

Email: boumediene.nedjar@ifsttar.fr
} 
starting from that choice. On the other hand, in $^{32}$, the authors did not take into account the magnetization neither for the instantaneous (equilibrium) nor for the elastically relaxed (non-equilibrium) parts of the response, reducing the magneto-viscoelasticy to its simplest form. However, the field theory developed in this paper fully couples the magnetic fields with finite viscoelasticity for compressible as well as for incompressible magnetizable materials in the spirit of an analogous development made in ${ }^{33}$ for finite strain electro-viscoelastic soft materials.

The coupling with magnetostatics is integrated within the framework of continuum thermodynamics for the correct setting of the whole set of constitutive laws together with the characterization of the dissipative phenomena through evolution equations. Herein, only isotropic MAPs are considered. In the formulation developed in this work, the magnetic induction vector is chosen to be the main independent variable for the magnetic part of the problem and, consequently, we show that the magnetic field becomes then implicitly decomposed additively into an equilibrium and a non-equilibrium parts. A simple model example is detailed for demonstrative purposes to highlight some phenomena qualitatively with parametric studies.

An outline of the remainder of this paper is as follows. In Section 2, we recall the governing equations of mechanical balance together with the specialized versions of Maxwell's equations in magnetostatics. Both of the equivalent spatial and material descriptions are considered. Then, in Section 3, the magnetoviscoelastic coupling is developed in detail and studied in Section 4 with parametric studies. Finally, conclusions and perspectives are drawn in Section 5.

Notations: Throughout the paper, bold face characters refer to second- or fourth-order tensorial quantities. In particular, 1 denotes the second-order identity tensor with components $\delta_{i j}\left(\delta_{i j}\right.$ being the Kronecker delta), and $\boldsymbol{I}$ is the fourth-order unit tensor of components $I_{i j k l}=\frac{1}{2}\left(\delta_{i k} \delta_{j l}+\delta_{i l} \delta_{j k}\right)$. The notation $(.)^{T}$ is used for the transpose operator and the double dot symbol ' $:$ ' is used for double tensor contraction, i.e. for any second-order tensors $\boldsymbol{A}$ and $\boldsymbol{B}, \boldsymbol{A}: \boldsymbol{B}=\operatorname{tr}\left[\boldsymbol{A} \boldsymbol{B}^{T}\right]=A_{i j} B_{i j}$ where, unless specified, summation on repeated indices is always assumed. The notation $\otimes$ stands for the tensorial product. In components, one has $(\boldsymbol{A} \otimes \boldsymbol{B})_{i j k l}=A_{i j} B_{k l}$, and for any two vectors $\mathbb{U}$ and $\mathbb{V},(\mathbb{U} \otimes \mathbb{V})_{i j}=U_{i} V_{j}$. Herein, the double-striked characters will exclusively be used for vector fields related to the magnetic part of the problem, e.g. $\mathfrak{b}, \mathbb{B}, \mathbb{h}, \mathbb{M} \ldots$. Finally, the dot notation will always designate the material time derivative, i.e. $(\vdots) \equiv d(\cdot) / d t$.

\section{Basic equations}

When undeformed, unstressed, and in the absence of magnetic fields, the magnetically sensitive body occupies the reference configuration $\mathcal{B}_{0}$ with boundary $\partial \mathcal{B}_{0}$. We identify a material particle by its position vector in the reference configuration, $\boldsymbol{X} \in \mathcal{B}_{0}$, and trace its motion by its current position at time $t$ in 
the spatial configuration $\mathcal{B}_{t}, \boldsymbol{x}(\boldsymbol{X}, t) \in \mathcal{B}_{t}$. The deformation gradient is as usual defined as $\boldsymbol{F}=\nabla_{\boldsymbol{X}} \boldsymbol{x}$, where $\nabla_{\boldsymbol{X}}($.$) is the material gradient operator with respect to the reference coordinates \boldsymbol{X}$. The Jacobian of the transformation is given by the determinant $J=\operatorname{det} \boldsymbol{F}$, with the standard convention $J>0$.

\subsection{Magnetostatics equations}

The materials we consider being electrically non-conducting, the fields in magnetostatics are governed by the following specializations of Maxwell's equations in the absence of distributed currents:

$$
\operatorname{curl} \mathrm{h}=\mathbf{0}, \quad \operatorname{div} \mathrm{b}=0, \quad \text { in } \mathcal{B}_{t},
$$

where $\mathbb{h}$ and $\mathfrak{b}$ are respectively the magnetic field and magnetic induction vectors, both with respect to the spatial configuration. Inside the material, they are related by the standard relation

$$
\mathfrak{b}=\mu_{0}(\mathfrak{h}+\mathrm{m})
$$

where the field vector $\mathrm{m}$ is the spatial magnetization. The constant $\mu_{0}$ is the magnetic permeability of vacuum. Note that in vacuo: $\mathfrak{b}=\mu_{0} \mathfrak{h} . \operatorname{In}(1), \operatorname{curl}(\cdot)$ and $\operatorname{div}(\cdot)$ are respectively the rotational and divergence operators with respect to $x$. They are denoted by $\operatorname{Curl}(\cdot)$ and $\operatorname{Div}(\cdot)$ in the material configuration with respect to $\boldsymbol{X}$. At the boundary of the body, in the absence of surface currents, the magnetic field and the magnetic induction vectors must satisfy the continuity conditions:

$$
\boldsymbol{n} \times \llbracket \mathfrak{h} \rrbracket=\mathbf{0}, \quad \llbracket \mathrm{b} \rrbracket \cdot \boldsymbol{n}=0, \quad \text { on } \partial \mathcal{B}_{t},
$$

where $\llbracket(\cdot) \rrbracket=(\cdot)_{\text {outside }}-(\cdot)_{\text {inside }}$ is the jump across the boundary $\partial \mathcal{B}_{t}$ of unit normal $\boldsymbol{n}$. Notice that the continuity conditions (3) are also valid across a discontinuity within the body, see also ${ }^{34}$.

Pull-back to the reference configuration gives the Lagrangian counterparts of the above laws (1):

$$
\operatorname{Curl} \mathbb{H}=\mathbf{0}, \quad \operatorname{Div} \mathbb{B}=0, \quad \text { in } \mathcal{B}_{0},
$$

for the Lagrangian-Eulerian interconnections

$$
\mathbb{H}=\boldsymbol{F}^{T} \mathfrak{h}, \quad \mathbb{B}=J \boldsymbol{F}^{-1} \mathfrak{b}, \quad \mathbb{M}=\boldsymbol{F}^{T} \mathbb{m} .
$$

With these relations, the material version of the standard relation (2) is given by

$$
\mathbb{B}=\mu_{0} J C^{-1}(\mathbb{H}+\mathbb{M})
$$


where $\boldsymbol{C}=\boldsymbol{F}^{T} \boldsymbol{F}$ is the right Cauchy-Green tensor which, otherwise, is a strain measure for the deformable solid. The counterpart of the continuity conditions (3), this time for $\mathbb{H}$ and $\mathbb{B}$, are

$$
\mathbf{N} \times \llbracket \mathbb{H} \rrbracket=\mathbf{0}, \quad \llbracket \mathbb{B} \rrbracket \cdot \mathbf{N}=0, \quad \text { on } \partial \mathcal{B}_{0},
$$

where $\boldsymbol{N}$ is the unit normal to the reference boundary $\partial \mathcal{B}_{0}$ that corresponds to $\boldsymbol{n}$ through Nanson's formula $\boldsymbol{n} \mathrm{d} a=J \boldsymbol{F}^{-T} \boldsymbol{N d} A$, where $\mathrm{d} a$ and $\mathrm{d} A$ are area elements on $\partial \mathcal{B}_{t}$ and $\partial \mathcal{B}_{0}$, respectively.

\subsection{Mechanical balance equations}

In quasi-statics, the spatial form of the mechanical equilibrium equation together with the boundary condition can take the form

$$
\begin{aligned}
\operatorname{div} \boldsymbol{\sigma}+\mathbb{f}_{m}+\rho \boldsymbol{f}=\mathbf{0} & & \text { in } \mathcal{B}_{t}, \\
\boldsymbol{\sigma} \boldsymbol{n}=\boldsymbol{t} & & \text { on } \partial \mathcal{B}_{t},
\end{aligned}
$$

where $\sigma$ is the Cauchy stress tensor, $\rho$ the current mass density, $f$ the mechanical body force per unit mass, and $\mathbb{1}_{m}$ is the magnetic body force per unit current volume. The traction force $t$ comprises both the mechanical traction per unit area of the boundary $\partial \mathcal{B}_{t}$, and an additional mechanical traction due to the magnetic effects. Notice that due to the magnetization, the spatial stress tensor $\sigma$ is in general non-symmetric. Nevertheless, we adopt herein the nowadays well known structure where the stress is augmented with terms arising from the magnetic body force, e.g. see $^{17}$ for details. The balance equation (8) is equivalently written as

$$
\begin{aligned}
\operatorname{div} \tilde{\boldsymbol{\sigma}}+\rho \boldsymbol{f} & =\mathbf{0} & & \text { in } \mathcal{B}_{t}, \\
\llbracket \tilde{\boldsymbol{\sigma}} \rrbracket \boldsymbol{n} & =\mathbf{0} & & \text { on } \partial \mathcal{B}_{t},
\end{aligned}
$$

in terms of the, this time symmetric, total Cauchy stress tensor $\widetilde{\sigma}$ defined as, see for example ${ }^{10,12}$ :

$$
\tilde{\boldsymbol{\sigma}}=\boldsymbol{\sigma}+\mu_{0}^{-1}\left[\mathfrak{b} \otimes \mathfrak{b}-\frac{1}{2} \mathfrak{b} . \mathfrak{b} \mathbf{1}\right]+\mathrm{m} . \mathfrak{b} \mathbf{1}-\mathrm{m} \otimes \mathfrak{b},
$$

where the second-to-fourth terms on the right-hand side of (10) come from the magnetic body force, here given by $\mathbb{f}_{m}=\left(\nabla_{\boldsymbol{x}} \mathfrak{b}\right)^{T} \mathrm{~m}$, see for example ${ }^{8,20}$. Here and henceforth, the notation $\nabla_{\boldsymbol{x}}(\cdot)$ refers to the spatial gradient operator with respect to the coordinates $\boldsymbol{x}$. The boundary condition $(9)_{2}$ is here equivalently written in the form of a jump condition.

Pull-back of the balance equation (9) to the reference configuration gives the following useful Lagrangian form

$$
\begin{aligned}
\operatorname{Div} \widetilde{\boldsymbol{P}}+\rho_{0} \boldsymbol{f} & =\mathbf{0} & & \text { in } \mathcal{B}_{0}, \\
\llbracket \widetilde{\boldsymbol{P}} \rrbracket \boldsymbol{N} & =\mathbf{0} & & \text { on } \partial \mathcal{B}_{0},
\end{aligned}
$$


in terms of the total first Piola-Kirchhoff stress tensor $\widetilde{\boldsymbol{P}}=J \widetilde{\boldsymbol{\sigma}} \boldsymbol{F}^{-T}$ and the reference density $\rho_{0}=J \rho$. In particular for later use, the expression of $\widetilde{\boldsymbol{P}}$ deduced from (10) is given by

$$
\begin{aligned}
\widetilde{\boldsymbol{P}}=\boldsymbol{P} & +\mu_{0}^{-1} J^{-1}\left[\boldsymbol{F} \mathbb{B} \otimes \mathbb{B}-\frac{1}{2} \boldsymbol{C}: \mathbb{B} \otimes \mathbb{B} \boldsymbol{F}^{-T}\right] \\
& +\mathbb{M} \cdot \mathbb{B} \boldsymbol{F}^{-T}-\boldsymbol{F}^{-T} \mathbb{M} \otimes \mathbb{B}
\end{aligned}
$$

where $\boldsymbol{P}=J \boldsymbol{\sigma} \boldsymbol{F}^{-T}$ and, among others, use has been made of the relations $(5)_{2}$ and $(5)_{3}$.

\section{Continuum thermodynamics and constitutive laws}

The above governing equations need to be supplemented with constitutive relations. For the magnetic part of the problem, we have at our disposal the three magnetic field vectors $\mathbb{B}, \mathbb{H}, \mathbb{M}$, with the connection (6). Any one of these can be used as the independent magnetic variable in the formulation of a constitutive law together with the characterization of the viscoelastic dissipation. In this work, we consider a formulation based on the use of the magnetic induction vector $\mathbb{B}$ that we detail herein under the assumption of isothermal conditions. The balance of energy is written in the local form as, see ${ }^{8,10}$ for example,

$$
\rho \dot{e}=\operatorname{div}\left(\boldsymbol{\sigma}^{T} \boldsymbol{v}\right)+\rho \boldsymbol{f} . \boldsymbol{v}+w_{m},
$$

where $e$ is the specific internal energy, per unit mass, $\boldsymbol{v}$ is the spatial velocity, and $w_{m}$ is the magnetic power given by $w_{m}=\mathbb{H}_{m} \cdot \boldsymbol{v}-\mathrm{m} . \dot{\mathrm{b}}$. Using the mechanical balance (8), we get the reduced form

$$
\rho \dot{e}=\boldsymbol{\sigma}: \nabla_{\boldsymbol{x}} \boldsymbol{v}-\mathrm{m} \cdot \dot{\mathrm{b}}
$$

where the spatial velocity gradient $\nabla_{\boldsymbol{x}} \boldsymbol{v}$ is related to the deformation gradient through the well known kinematic formula $\nabla_{\boldsymbol{x}} \boldsymbol{v}=\dot{\boldsymbol{F}} \boldsymbol{F}^{-1}$.

The second law of thermodynamics postulates the positiveness of the entropy production. Denoting by $s$ the specific entropy per unit mass, we write $\dot{s} \geq 0$ and, for isothermal processes, the dissipation $\mathcal{D}$ is given by

$$
\mathcal{D}=\rho T_{0} \dot{s} \geq 0
$$

where $T_{0}$ is the fixed absolute temperature.

Now defining the specific free energy function $\hat{\psi}$ as $\hat{\psi}=e-T_{0} s$, using this latter together with (14) into (15) gives the classical form,

$$
\mathcal{D}=\boldsymbol{\sigma} \boldsymbol{F}^{-T}: \dot{\boldsymbol{F}}-\mathrm{m} \cdot \dot{\mathrm{b}}-\rho \dot{\hat{\psi}} \geq 0
$$


Notice that in magneto-elasticity with a free energy function based on the use of the Eulerian magnetic induction as $\hat{\psi}(\boldsymbol{F}, \mathfrak{b})$, this leads to the well-known constitutive equations,

$$
\boldsymbol{\sigma}=\rho \frac{\partial \hat{\psi}}{\partial \boldsymbol{F}} \boldsymbol{F}^{T}, \quad \mathrm{~m}=-\rho \frac{\partial \hat{\psi}}{\partial \mathfrak{b}}
$$

for the Cauchy stress tensor and the magnetization vector. However, as the Lagrangian form is better suited for the following thermodynamic developments, inequality (16) must be pull-back to the reference configuration. Thus, we may regard the specific free energy function $\hat{\psi}$, equivalently, as a function of $\boldsymbol{F}$ and $\mathbb{B}$, and we introduce the notation $\psi$ for this purpose as

$$
\psi(\boldsymbol{F}, \mathbb{B} \ldots) \equiv \hat{\psi}\left(\boldsymbol{F}, J^{-1} \boldsymbol{F} \mathbb{B} \ldots\right)
$$

where the dots mean further arguments that will eventually be introduced later on for the purpose of viscoelastic modelling. Thereby, using the relations $\boldsymbol{\sigma} \boldsymbol{F}^{-T}=J^{-1} \boldsymbol{P}, \rho=J^{-1} \rho_{0}$ and

$$
\mathrm{m} . \dot{\mathrm{b}}=J^{-1} \mathbb{M} \cdot \dot{\mathbb{B}}-J^{-1} \mathbb{M} \cdot \mathbb{B} \boldsymbol{F}^{-T}: \dot{\boldsymbol{F}}+J^{-1} \boldsymbol{F}^{-T} \mathbb{M} \otimes \mathbb{B}: \dot{\boldsymbol{F}}
$$

where use has been made of $(5)_{2}$ and $(5)_{3}$ together with the well known kinematic relation $\dot{J}=J \boldsymbol{F}^{-T}$ : $\dot{\boldsymbol{F}}$, the inequality (16) becomes,

$$
\mathcal{D}=\left(\boldsymbol{P}+\mathbb{M} \cdot \mathbb{B} \boldsymbol{F}^{-T}-\boldsymbol{F}^{-T} \mathbb{M} \otimes \mathbb{B}\right): \dot{\boldsymbol{F}}-\mathbb{M} \cdot \dot{\mathbb{B}}-\rho_{0} \dot{\psi} \geq 0 .
$$

This latter can further be simplified by introducing the augmented free energy function $\Omega$ per unit reference volume defined as, see for example ${ }^{11}$,

$$
\Omega(\boldsymbol{F}, \mathbb{B} \ldots)=\rho_{0} \psi+\frac{1}{2} \mu_{0}^{-1} J^{-1} \boldsymbol{C}: \mathbb{B} \otimes \mathbb{B},
$$

which, replaced into (18), transforms this latter into the more convenient form

$$
\mathcal{D}=\widetilde{\boldsymbol{P}}: \dot{\boldsymbol{F}}+\mathbb{H} \cdot \dot{\mathbb{B}}-\dot{\Omega} \geq 0,
$$

where use has been made of the definition (12) for the total first Piola-Kirchhoff stress tensor $\widetilde{\boldsymbol{P}}$ and of the relation (6) for the Lagrangian magnetic induction vector $\mathbb{B}$. Among others, observe that, in this form, the conjugate character between $\mathbb{H}$ and $\mathbb{B}$ replaces the one between $\mathbb{M}$ and $\mathbb{B}$ in the former form (18). For similarities, see also ${ }^{16}$ for developments in magneto-poromechanics. 
The equal sign in (20) takes place only for non-dissipative processes, i.e. magneto-elasticity. In this case the thermodynamic equilibrium state is fully determined by the deformation gradient and the magnetic induction. For a viscoelastic material, however, the free energy in a non-equilibrium state differs from that in an equilibrium state. To distinguish between them, we introduce the equilibrium free energy $\psi_{\text {eq }}(\boldsymbol{F}, \mathbb{B})$ so that from $(20)$ in the case of an equal sign we get by thermodynamic arguments, see ${ }^{35}$,

$$
\widetilde{\boldsymbol{P}}_{\mathrm{eq}}=\frac{\partial \Omega_{\mathrm{eq}}}{\partial \boldsymbol{F}}, \quad \mathbb{H}_{\mathrm{eq}}=\frac{\partial \Omega_{\mathrm{eq}}}{\partial \mathbb{B}}
$$

where $\widetilde{\boldsymbol{P}}_{\text {eq }}$ and $\mathbb{H}_{\text {eq }}$ are the total first Piola-Kirchhoff stress tensor and the Lagrangian magnetic field vector in an equilibrium state, and where $\Omega_{\mathrm{eq}}$ is the augmented volumetric free energy function at thermodynamic equilibrium defined as, see (19),

$$
\Omega_{\mathrm{eq}}(\boldsymbol{F}, \mathbb{B})=\rho_{0} \psi_{\mathrm{eq}}(\boldsymbol{F}, \mathbb{B})+\frac{1}{2} \mu_{0}^{-1} J^{-1} \boldsymbol{C}: \mathbb{B} \otimes \mathbb{B}
$$

The simple structures of the constitutive formulas (21) are exactly those derived for magneto-elastic modelling, see for example ${ }^{17,36}$.

\subsection{Kinematic assumptions and constitutive laws}

The viscoelastic response is here captured by means of the nowadays well-accepted multiplicative split of the deformation gradient into an elastically relaxing part $\boldsymbol{F}^{\mathrm{e}}$ and a viscous part $\boldsymbol{F}^{\mathrm{v}}$ as illustrated in Fig. 1, for instance, see $29,30,37$ among others:

$$
\boldsymbol{F}=\boldsymbol{F}^{\mathrm{e}} \boldsymbol{F}^{\mathrm{v}}
$$

Now motivated by the decomposition of the energy in the purely mechanical theory, the nonequilibrium free energy that we denote here by $\psi_{\text {neq }}$ is the difference between the total free energy $\psi$ and that of an equilbrium state $\psi_{\text {eq }} ; \psi-\psi_{\text {eq }} \equiv \psi_{\text {neq. }}$. Herein, this latter is associated with the elastically relaxing part of the deformation gradient $\boldsymbol{F}^{\mathrm{e}}$. It can moreover depend on the magnetic induction which, otherwise, is assumed to equilibrate much faster than deformation:

$$
\psi_{\text {neq }} \equiv \psi_{\text {neq }}\left(\boldsymbol{F}^{\mathrm{e}}=\boldsymbol{F} \boldsymbol{F}^{\mathrm{v}^{-1}}, \overline{\mathbb{B}}=J^{\mathrm{v}^{-1}} \boldsymbol{F}^{\mathrm{v}} \mathbb{B}\right)
$$

where $\overline{\mathbb{B}}$ is the push-forward with $\boldsymbol{F}^{\mathrm{v}}$ of the Lagrangian magnetic induction vector $\mathbb{B}$ to the intermediate configuration, see the illustration on the sketch of Fig. 1. It is equivalently the pull-back with $\boldsymbol{F}^{\mathrm{e}}$ of the spatial magnetic induction vector $\mathfrak{b}$. Here $J^{\mathrm{v}}=\operatorname{det} \boldsymbol{F}^{\mathrm{v}}$ and $J^{\mathrm{e}}=\operatorname{det} \boldsymbol{F}^{\mathrm{e}}$ are the Jacobians of the viscous 


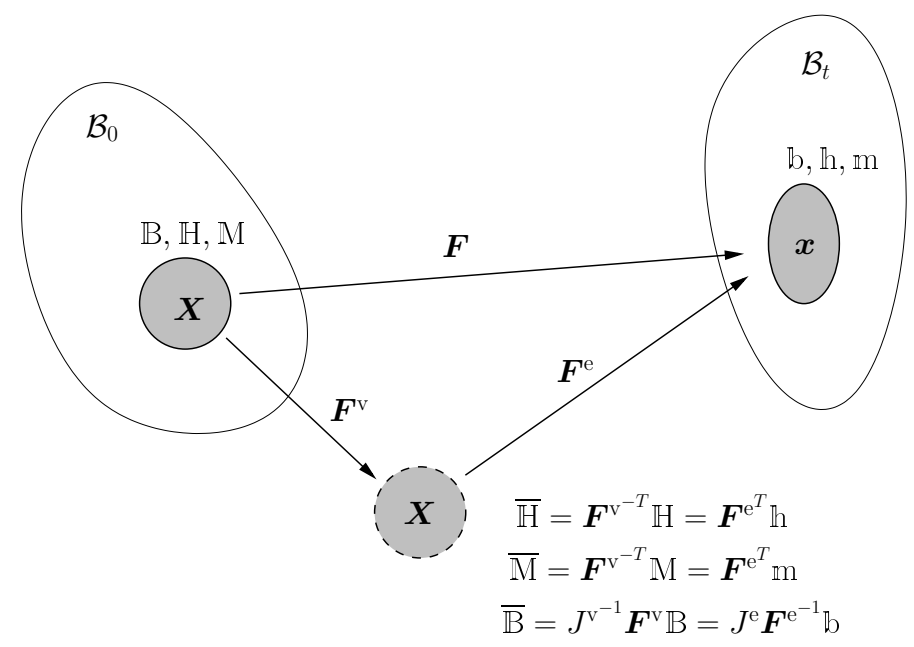

Figure 1. Illustration of the multiplicative decomposition $\boldsymbol{F}=\boldsymbol{F}^{\mathrm{e}} \boldsymbol{F}^{\mathrm{v}}$ and transport of the magnetic field vectors on the intermediate configuration.

and the elastically relaxing parts of the deformation gradient. The precise forms of the above constitutive functions $\psi_{\text {eq }}$ and $\psi_{\text {neq }}$ are dictated by invariance and material symmetry that will be discussed below.

With the additive structure of $\psi$, we choose for convenience to re-cast the augmented free energy $\Omega$, Eq. (19), into the following form

$$
\Omega=\Omega_{\mathrm{eq}}+\rho_{0} \psi_{\text {neq }},
$$

where $\Omega_{\text {eq }}$ is the augmented form already defined in (22). The constitutive equations are given by the derivatives of $\Omega$ as,

$$
\widetilde{\boldsymbol{P}}=\frac{\partial \Omega}{\partial \boldsymbol{F}}=\widetilde{\boldsymbol{P}}_{\mathrm{eq}}+\underbrace{\rho_{0} \frac{\partial \psi_{\text {neq }}}{\partial \boldsymbol{F}^{\mathrm{e}}} \boldsymbol{F}^{\mathbf{v}^{-T}}}_{\boldsymbol{P}_{\text {neq }}}, \quad \mathbb{H}=\frac{\partial \Omega}{\partial \mathbb{B}}=\mathbb{H}_{\mathrm{eq}}+\underbrace{J^{\mathbf{v}^{-1}} \boldsymbol{F}^{\mathbf{v}^{T}} \rho_{0} \frac{\partial \psi_{\text {neq }}}{\partial \overline{\mathbb{B}}}}_{\mathbb{H}_{\text {neq }}},
$$


where $\widetilde{\boldsymbol{P}}_{\text {eq }}$ and $\mathbb{H}_{\text {eq }}$ have been defined in (21). The remainder of the inequality (20) that governs the evolution of the internal variable, here $\boldsymbol{F}^{\mathrm{v}}$, becomes

$$
\mathcal{D}=\rho_{0}\left(\boldsymbol{F}^{\mathrm{e}^{T}} \frac{\partial \psi_{\text {neq }}}{\partial \boldsymbol{F}^{\mathrm{e}}}-\frac{\partial \psi_{\text {neq }}}{\partial \overline{\mathbb{B}}} \otimes \overline{\mathbb{B}}+\overline{\mathbb{B}} \cdot \frac{\partial \psi_{\text {neq }}}{\partial \overline{\mathbb{B}}} \mathbf{1}\right): \boldsymbol{l}^{\mathrm{v}} \geq 0
$$

where $\boldsymbol{l}^{\mathrm{v}}=\dot{\boldsymbol{F}}^{\mathrm{v}} \boldsymbol{F}^{\mathrm{v}}{ }^{-1}$ is the left-rate of the viscous part of the deformation gradient, which is in general non-symmetric.

As expected, the total stress tensor is additively split into a part that is in thermodynamic equilibrium, herein written in the augmented form $\widetilde{\boldsymbol{P}}_{\text {eq }}$, and an over-stress $\boldsymbol{P}_{\text {neq }}$ that is thermodynamically not in equilibrium. For convenience, we adopt the same notation for the magnetic field vector $\mathbb{H}$; with subscript notation $(\cdot)_{\text {eq }}$ for the term derived from the part $\Omega_{\mathrm{eq}}$ of the augmented free energy, and subscript notation (.) neq for the term derived from the part $\rho_{0} \psi_{\text {neq. }}$. This notation will henceforth be used for all the magnetic fields. For instance, one has then

$$
\mathbb{h}=\boldsymbol{F}^{-T} \mathbb{H}=\underbrace{\boldsymbol{F}^{-T} \frac{\partial \Omega_{\mathrm{eq}}}{\partial \mathbb{B}}}_{\mathbb{h}_{\mathrm{eq}}}+\underbrace{J^{\mathrm{v}^{-1}} \boldsymbol{F}^{\mathrm{e}^{-T}} \rho_{0} \frac{\partial \psi_{\mathrm{neq}}}{\partial \overline{\mathbb{B}}}}_{\mathbb{h}_{\text {neq }}},
$$

for the spatial magnetic field vector, and

$$
\overline{\mathbb{H}}=\boldsymbol{F}^{\mathbf{v}^{-T}} \mathbb{H} \equiv \boldsymbol{F}^{\mathrm{e}^{T}} \mathfrak{h}=\underbrace{\boldsymbol{F}^{\mathrm{v}^{-T}} \frac{\partial \Omega_{\mathrm{eq}}}{\partial \mathbb{B}}}_{\overline{\mathbb{H}}_{\mathrm{eq}}}+\underbrace{J^{\mathrm{v}^{-1}} \rho_{0} \frac{\partial \psi_{\mathrm{neq}}}{\partial \overline{\mathbb{B}}}}_{\overline{\mathbb{H}}_{\mathrm{neq}}},
$$

when transported to the intermediate configuration.

Now for objectivity reasons, $\Omega_{\text {eq }}$ depends on the deformation gradient $\boldsymbol{F}$ only through the right Cauchy-Green tensor $\boldsymbol{C}$, and for symmetry reasons, it depends on the magnetic induction vector $\mathbb{B}$ only through the tensor product $\mathbb{B} \otimes \mathbb{B}$. Likewise, the part $\psi_{\text {neq }}$ depends on $\boldsymbol{F}^{\mathrm{e}}$ only through the elastically relaxing right Cauchy-Green tensor $\boldsymbol{C}^{\mathrm{e}}=\boldsymbol{F}^{\mathrm{e}^{T}} \boldsymbol{F}^{\mathrm{e}} \equiv \boldsymbol{F}^{\mathrm{v}^{-T}} \boldsymbol{C} \boldsymbol{F}^{\mathrm{v}^{-1}}$, and on $\overline{\mathbb{B}}$ only through the tensor product $\overline{\mathbb{B}} \otimes \overline{\mathbb{B}} \equiv J^{\mathrm{v}^{-2}} \boldsymbol{F}^{\mathrm{v}} \mathbb{B} \otimes \mathbb{B} \boldsymbol{F}^{\mathrm{v}^{T}}$, both defined in the intermediate configuration, i.e.

$$
\Omega \equiv \Omega_{\mathrm{eq}}(\boldsymbol{C}, \mathbb{B} \otimes \mathbb{B})+\rho_{0} \psi_{\text {neq }}\left(\boldsymbol{C}^{\mathrm{e}}, \overline{\mathbb{B}} \otimes \overline{\mathbb{B}}\right)
$$


The above stress constitutive relation $(26)_{1}$ is then equivalently written as

$$
\widetilde{\boldsymbol{S}} \equiv \boldsymbol{F}^{-1} \widetilde{\boldsymbol{P}}=\underbrace{2 \frac{\partial \Omega_{\mathrm{eq}}}{\partial \boldsymbol{C}}}_{\widetilde{\boldsymbol{S}}_{\mathrm{eq}}}+\underbrace{\boldsymbol{F}^{\mathrm{v}^{-1}} \underbrace{2 \rho_{0} \frac{\partial \psi_{\mathrm{neq}}}{\partial \boldsymbol{C}^{\mathrm{e}}}}_{\overline{\boldsymbol{S}}_{\text {neq }}} \boldsymbol{F}^{\mathrm{v}^{-T}}}_{\boldsymbol{S}_{\text {neq }}},
$$

for the total second Piola-Kirchhoff-type, where $\overline{\boldsymbol{S}}_{\text {neq }}=\boldsymbol{F}^{\mathrm{v}} \boldsymbol{S}_{\text {neq }} \boldsymbol{F}^{\mathrm{v}}$ is the push-forward of its nonequilibrium part $\boldsymbol{S}_{\text {neq }}$ to the intermediate configuration. The reduced dissipation (27) can alternatively be written as

$$
\mathcal{D}=\left(\boldsymbol{C}^{\mathrm{e}} \overline{\boldsymbol{S}}_{\text {neq }}-J^{\mathrm{v}} \overline{\mathbb{H}}_{\text {neq }} \otimes \overline{\mathbb{B}}+J^{\mathrm{v}} \overline{\mathbb{H}}_{\text {neq }} \cdot \overline{\mathbb{B}} \mathbf{1}\right): \operatorname{sym}\left[\boldsymbol{l}^{\mathrm{v}}\right] \geq 0,
$$

where sym [.] is the symmetric part operator of a second-order tensor. In fact, we will show later on that the above term between parentheses in (32) is indeed symmetric.

\subsection{Characterization of the viscoelastic evolution}

A local evolution equation must now be characterized such that the above inequality (32) is satisfied for any admissible process. One way to do so is to rewrite this latter in the equivalent spatial form by exploiting the kinematic relations

$$
\boldsymbol{b}^{\mathrm{e}}=\boldsymbol{F}^{\mathrm{e}} \boldsymbol{F}^{\mathrm{e}^{T}} \equiv \boldsymbol{F} \boldsymbol{C}^{\mathrm{v}^{-1}} \boldsymbol{F}^{T}, \quad \text { with } \boldsymbol{C}^{\mathrm{v}}=\boldsymbol{F}^{\mathrm{v}^{T}} \boldsymbol{F}^{\mathrm{v}}
$$

where $\boldsymbol{b}^{\mathrm{e}}$ is the elastically relaxing left Cauchy-Green tensor and $\boldsymbol{C}^{\mathrm{v}}$ is the viscous right Cauchy-Green tensor. One obtains the following well-known relation:

$$
\operatorname{sym}\left[\boldsymbol{l}^{\mathrm{v}}\right]=\boldsymbol{F}^{\mathrm{e}^{-1}}\left(-\frac{1}{2} £_{v} \boldsymbol{b}^{\mathrm{e}}\right) \boldsymbol{F}^{\mathrm{e}^{-T}}
$$

where $£_{v} \boldsymbol{b}^{\mathrm{e}}=\boldsymbol{F} \dot{\boldsymbol{C}}^{\mathrm{v}^{-1}} \boldsymbol{F}^{T}$ is the Lie derivative of $\boldsymbol{b}^{\mathrm{e}}$, see for example ${ }^{38,39}$ for details. Then, replacing (34) into (32) gives the following spatial version:

$$
\mathcal{D}=\left(\tau_{\text {neq }}-J h_{\text {neq }} \otimes \mathfrak{b}+J h_{\text {neq }} \cdot \mathfrak{b} \mathbf{1}\right) \boldsymbol{b}^{\mathrm{e}^{-1}}:\left(-\frac{1}{2} £_{v} \boldsymbol{b}^{\mathrm{e}}\right) \geq 0
$$

where use has been made of the push-forward transformations $\boldsymbol{\tau}_{\text {neq }}=\boldsymbol{F}^{\mathrm{e}} \overline{\boldsymbol{S}}_{\text {neq }} \boldsymbol{F}^{\mathrm{e}^{T}}, J^{\mathrm{e}} \mathrm{b}=\boldsymbol{F}^{\mathrm{e}} \overline{\mathbb{B}}$ and $\mathfrak{h}_{\text {neq }}=\boldsymbol{F}^{\mathrm{e}^{-T}} \overline{\mathbb{H}}_{\text {neq }}$, together with the kinematic relation $J=J^{\mathrm{e}} J^{\mathrm{v}}$. 
Among many possibilities, the following general form can be adopted for the evolution equation:

$$
-\frac{1}{2} £_{v} \boldsymbol{b}^{\mathrm{e}}=\mathbf{M}:\left(\left(\boldsymbol{\tau}_{\text {neq }}-J \mathrm{~h}_{\text {neq }} \otimes \mathfrak{b}+J \mathfrak{h}_{\text {neq }} \cdot \mathfrak{b} \mathbf{1}\right) \boldsymbol{b}^{\mathrm{e}^{-1}}\right)
$$

where the fourth-order mobility tensor $\mathbf{M}$ is positive definite so that the inequality (35), or equivalently (32), is satisfied automatically. This form is indeed often used in practice, see for example ${ }^{26,40,41}$ for similarities. In general, the mobility tensor may depend on various state variables. A possible physical meaning of the evolution law (36) is that one type of polymer chains relaxes in time and dissipates energy. This can be pictured by a dashpot in series with a spring. In the current state, the stress acting in the spring drives the viscous deformation in the dashpot, here the viscous strain tensor $\boldsymbol{C}^{\mathrm{v}}$ related to $\boldsymbol{b}^{\mathrm{e}}$ through $(33)_{1}$. The key observation in this work is that the stress acting in the spring is herein coupled with stresses emanating from the magnetic effects on the intermediate configuration if the magnetic induction is accounted for in the expression of $\psi_{\text {neq }}$, see (24).

\subsection{A modelling example}

Being isotropic functions of their respective arguments, each part of the free energy function $\psi$ depends at most on a collection of six irreducible invariants. For the part related to the thermodynamic equilibrium $\psi_{\text {eq }}$, and hence to $\Omega_{\text {eq }}$ by $(22)$, it depends on

$$
\begin{gathered}
I_{1}=C: \mathbf{1}, \quad I_{2}=\frac{1}{2}\left(I_{1}^{2}-C: C\right), \quad I_{3}=\operatorname{det} \boldsymbol{C}=J^{2}, \\
I_{4}=\mathbb{B} \cdot \mathbb{B}, \quad I_{5}=C: \mathbb{B} \otimes \mathbb{B}, \quad \text { and } I_{6}=C^{2}: \mathbb{B} \otimes \mathbb{B},
\end{gathered}
$$

where, for the double tensor contraction, we recall that we use the convention stated in the Notations's paragraph of the introductory Section 1 , for example $I_{5}=C: \mathbb{B} \otimes \mathbb{B}=C_{K L} B_{K} B_{L}=F_{i K} F_{i L} B_{K} B_{L}$.

Likewise, the non-equilibrium part $\psi_{\text {neq }}$ depends at most on

$$
\begin{gathered}
\bar{I}_{1}=C^{\mathrm{e}}: \mathbf{1}, \quad \bar{I}_{2}=\frac{1}{2}\left(\bar{I}_{1}^{2}-\boldsymbol{C}^{\mathrm{e}}: \boldsymbol{C}^{\mathrm{e}}\right), \quad \bar{I}_{3}=\operatorname{det} \boldsymbol{C}^{\mathrm{e}}=J^{\mathrm{e}^{2}}, \\
\bar{I}_{4}=\overline{\mathbb{B}} \cdot \overline{\mathrm{B}}, \quad \bar{I}_{5}=\boldsymbol{C}^{\mathrm{e}}: \overline{\mathbb{B}} \otimes \overline{\mathbb{B}}, \quad \text { and } \bar{I}_{6}=\boldsymbol{C}^{\mathrm{e}^{2}}: \overline{\mathbb{B}} \otimes \overline{\mathbb{B}},
\end{gathered}
$$

see for example ${ }^{42}$ for details on the topic of invariance. The first three invariants in each set (37) and (38) are classical in isotropic viscoelasticity. The other ones arise from the magneto-mechanics coupling in the thermodynamically equilibrated as well as non-equilibrated states for the most general case. 
The following general forms for the parts $\Omega_{\text {eq }}$ and $\psi_{\text {neq }}$ can be adopted as a modelling example:

$$
\begin{aligned}
& \Omega_{\text {eq }}=\rho_{0} \psi_{\text {eq }}^{\prime}\left(I_{1}, I_{2}, I_{3}\right)+\underbrace{c_{1} I_{4}+c_{2} I_{5}+c_{3} I_{6}}_{\text {magnetic coupling }}+\underbrace{\frac{1}{2} \mu_{0}^{-1} J^{-1} \boldsymbol{C}: \mathbb{B} \otimes \mathbb{B}}_{\text {augmentation }}, \\
& \rho_{0} \psi_{\text {neq }}=\rho_{0} \psi_{\text {neq }}^{\prime}\left(\bar{I}_{1}, \bar{I}_{2}, \bar{I}_{3}\right)+\underbrace{c_{4} \bar{I}_{4}+c_{5} \bar{I}_{5}+c_{6} \bar{I}_{6}}_{\text {magnetic coupling }},
\end{aligned}
$$

where $c_{1}, c_{2}, \ldots, c_{6}$ are material parameters relative to the magnetic coupling. The first volumetric terms $\rho_{0} \psi_{\text {eq }}^{\prime}$ and $\rho_{0} \psi_{\text {neq }}^{\prime}$ are related to the purely mechanical part. Furthermore, with the form $(39)_{2}$, one can easily check that the term between parentheses in (32) is symmetric.

Applying the above forms (39) to Eq. (31), we obtain the following relation for the total stress tensor of the Kirchhoff-type $\widetilde{\boldsymbol{\tau}}=\boldsymbol{F} \widetilde{\boldsymbol{S}} \boldsymbol{F}^{T} \equiv J \widetilde{\boldsymbol{\sigma}}$ :

$$
\begin{aligned}
\widetilde{\boldsymbol{\tau}}=\tau_{\text {eq }}^{\prime}+\tau_{\text {neq }}^{\prime} & +2 c_{2} J^{2} \mathfrak{b} \otimes \mathfrak{b}+2 c_{3} J^{2}\{\mathfrak{b} \otimes \mathfrak{b} \boldsymbol{b}+\boldsymbol{b} \mathfrak{b} \otimes \mathfrak{b}\} \\
& +2 c_{5} J^{\mathrm{e}^{2}} \mathrm{~b} \otimes \mathfrak{b}+2 c_{6} J^{\mathrm{e}^{2}}\left\{\mathfrak{b} \otimes \mathfrak{b} \boldsymbol{b}^{\mathrm{e}}+\boldsymbol{b}^{\mathrm{e}} \mathrm{b} \otimes \mathfrak{b}\right\} \\
& +\mu_{0}^{-1} J\left\{\mathrm{~b} \otimes \mathfrak{b}-\frac{1}{2} \mathfrak{b} . \mathfrak{b} \mathbf{1}\right\},
\end{aligned}
$$

where we have introduced for convenience the notations

$$
\boldsymbol{\tau}_{\text {eq }}^{\prime}=2 \rho_{0} \boldsymbol{F} \frac{\partial \psi_{\text {eq }}^{\prime}}{\partial \boldsymbol{C}} \boldsymbol{F}^{T}, \quad \text { and } \quad \boldsymbol{\tau}_{\text {neq }}^{\prime}=2 \rho_{0} \boldsymbol{F}^{\mathrm{e}} \frac{\partial \psi_{\text {neq }}^{\prime}}{\partial \boldsymbol{C}^{\mathrm{e}}} \boldsymbol{F}^{\mathrm{e}^{T}},
$$

for the purely mechanical parts. Here use has been made of the transformations $(5)_{2}$ and $J^{\mathrm{e}} \mathfrak{b}=\boldsymbol{F}^{\mathrm{e}} \overline{\mathbb{B}}$ for the spatial induction vector $\mathfrak{b}$, and $\boldsymbol{b}=\boldsymbol{F} \boldsymbol{F}^{T}$ is the well known (spatial) left Cauchy-Green tensor. The equilibrium part of the total stress in (41) agrees with the most general form established in ${ }^{10}$ for the case of compressible magnetoelasticity. One can also observe that a magnetostatic contribution appears in the inelastic stress through the material parameters $c_{5}$ and $c_{6}$.

For the spatial magnetic field vector, we have from (39), (26) $)_{2}$ and (28):

$$
\begin{aligned}
& \mathrm{h}=\underbrace{2 c_{1} J \boldsymbol{b}^{-1} \mathfrak{b}+2 c_{2} J \mathfrak{b}+2 c_{3} J \boldsymbol{b} \mathfrak{b}}_{=-\mathrm{m}_{\mathrm{eq}}}
\end{aligned}
$$

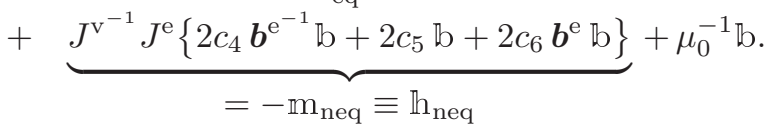


Comparing the latter expressions (42) with the corresponding standard relation (2), one concludes that $c_{1}, c_{2}, \ldots, c_{6}$ are in fact the magnetization parameters, among which, $c_{4}, c_{5}$ and $c_{6}$ are related to the intermediate configutation.

Now for the purpose of demonstration, a simple choice for the mobility tensor in (36) could be $\mathbf{M}=1 / \eta \boldsymbol{I}$ where $\eta>0$ is a viscosity parameter of dimension $\left[\mathrm{Nm}^{-2} \mathrm{~s}\right]$ that can be assumed constant in a first approach. The evolution equation (36) would then become

$$
\begin{aligned}
-\frac{1}{2} £_{v} \boldsymbol{b}^{\mathrm{e}}=\frac{1}{\eta} & \left\{\boldsymbol{\tau}_{\text {neq }}^{\prime} \boldsymbol{b}^{\mathrm{e}^{-1}}-2 c_{4} J^{\mathrm{e}^{2}} \boldsymbol{b}^{\mathrm{e}^{-1}} \mathrm{~b} \otimes \mathfrak{b} \boldsymbol{b}^{\mathrm{e}^{-1}}+2 c_{6} J^{\mathrm{e}^{2}} \mathrm{~b} \otimes \mathfrak{b}\right. \\
& \left.+2\left(c_{4} \bar{I}_{4}+c_{5} \bar{I}_{5}+c_{6} \bar{I}_{6}\right) \boldsymbol{b}^{\mathrm{e}^{-1}}\right\},
\end{aligned}
$$

after having replaced the expressions of $\tau_{\text {neq }}$ and $\mathfrak{h}_{\text {neq }}$ from Eqs. (40) and (42), respectively, and simplifying terms. Among others, notice that both sides of (43) are symmetric. However, it should be stressed that in general the viscosity parameter $\eta$ is not constant, e.g. see for example ${ }^{21,43}$.

Remark 1. If the energy dissipation due to the magnetization is negligible, then by setting $c_{4}=c_{5}=$ $c_{6}=0$, the model drastically simplifies as follows:

- The part of the the magnetic field vector relative to the intermediate configuration would vanish as $\mathrm{H}_{\text {neq }}=\mathrm{h}_{\text {neq }}=\mathbf{0}$.

- The over-stress would reduce to its purely mechanical part as $\tau_{\text {neq }}=\tau_{\text {neq }}^{\prime}$ for the Kirchoff-type stress tensor.

- The reduced dissipation (35) would take its classical form encountered in finite isotropic viscoelasticity.

- If, furthermore $c_{1}=c_{2}=c_{3}=0$, then the magnetization vector field vanishes as $\mathbb{M}=\mathbf{0}$. In this case the present modelling reduces to that of non-magnetizable magneto-viscoelasticity as developed in ${ }^{32}$.

\section{Homogeneous deformation and parametric studies}

In this section, we will construct a simple model to study some phenomena qualitatively. To this end, it remains now to specify the purely mechanical free energy functions $\psi_{\text {eq }}^{\prime}$ and $\psi_{\text {neq }}^{\prime}$ so that the augmented free energy function $\Omega$ will be completely defined, see (30) and (39). Here for the purpose of demonstration, we choose a compressible Neo-Hookean type modelling for both functions as

$$
\begin{aligned}
& \rho_{0} \psi_{\text {eq }}^{\prime}=\frac{3}{8} \kappa_{\text {eq }}\left(J^{\frac{4}{3}}+2 J^{-\frac{2}{3}}-3\right)+\frac{1}{2} \mu_{\text {eq }}\left(J^{-\frac{2}{3}} I_{1}-3\right), \\
& \rho_{0} \psi_{\text {neq }}^{\prime}=\frac{3}{8} \kappa_{\text {neq }}\left(J^{\mathrm{e}^{\frac{4}{3}}}+2 J^{\mathrm{e}^{-\frac{2}{3}}}-3\right)+\frac{1}{2} \mu_{\text {neq }}\left(J^{\mathrm{e}^{-\frac{2}{3}}} \bar{I}_{1}-3\right),
\end{aligned}
$$


where the first terms are related to the volumetric response with $\kappa_{\mathrm{eq}}$ and $\kappa_{\text {neq }}$ the equilibrium and nonequilibrium bulk moduli, respectively, while the second terms are related to the isochoric part of the response with $\mu_{\mathrm{eq}}$ and $\mu_{\mathrm{neq}}$ as the equilibrium and the non-equilibrium shear moduli, respectively, e.g. see also $^{44,45}$ for more details about the properties of the hyperelastic version of the model (44). With these forms, the purely mechanical parts of the Kirchhoff-type stress tensor are given by, see Eq. (40),

$$
\begin{aligned}
\tau_{\text {eq }}^{\prime} & =\frac{1}{2} \kappa_{\text {eq }}\left(J^{\frac{4}{3}}-J^{-\frac{2}{3}}\right) \mathbf{1}+\mu_{\text {eq }} \operatorname{dev}\left[J^{-\frac{2}{3}} \boldsymbol{b}\right], \\
\boldsymbol{\tau}_{\text {neq }}^{\prime} & =\frac{1}{2} \kappa_{\text {neq }}\left(J^{\mathrm{e}^{\frac{4}{3}}}-J^{\mathrm{e}^{-\frac{2}{3}}}\right) \mathbf{1}+\mu_{\text {neq }} \operatorname{dev}\left[J^{\mathrm{e}^{-\frac{2}{3}}} \boldsymbol{b}^{\mathrm{e}}\right],
\end{aligned}
$$

where $\operatorname{dev}[$.$] denotes the spatial deviatoric operator.$

As a simple model problem, we study the response of a cylindrical sample to applied magnetic induction along its axial direction $\vec{e}_{3}$, i.e. $\mathbb{B}(t)=B_{3}(t) \vec{e}_{3}$. For simplicity, we assume the deformation to be homogeneous. Let $\lambda_{1}, \lambda_{2}$ and $\lambda_{3}$ be the principal stretches along the three directions $\vec{e}_{1}, \vec{e}_{2}$ and $\vec{e}_{3}$, as the loading direction does not change in the history, the principal directions of the viscoelastic stretches coincide with those of the above total ones, and we will denote them as $\lambda_{1}^{\mathrm{v}}, \lambda_{2}^{\mathrm{v}}$ and $\lambda_{3}^{\mathrm{v}}$. Furthermore, here for symmetry reasons, we also have $\lambda_{2}=\lambda_{1}$ and $\lambda_{2}^{\mathrm{v}}=\lambda_{1}^{\mathrm{v}}$.

In components, the constitutive relation (40) for the total Kirchhoff-type stress tensor $\widetilde{\tau}=J \widetilde{\sigma}$ specializes to

$$
\begin{aligned}
J \widetilde{\sigma}_{11} & =\frac{1}{2} \kappa_{\mathrm{eq}}\left(\lambda_{1}^{\frac{8}{3}} \lambda_{3}^{\frac{4}{3}}-\lambda_{1}^{-\frac{4}{3}} \lambda_{3}^{-\frac{2}{3}}\right)+\frac{1}{3} \mu_{\mathrm{eq}}\left(\lambda_{1}^{\frac{2}{3}} \lambda_{3}^{-\frac{2}{3}}-\lambda_{1}^{-\frac{4}{3}} \lambda_{3}^{\frac{4}{3}}\right) \\
& +\frac{1}{2} \kappa_{\text {neq }}\left(\lambda_{1}^{\mathrm{e}^{\frac{8}{3}}} \lambda_{3}^{\mathrm{e}^{\frac{4}{3}}}-\lambda_{1}^{\mathrm{e}^{-\frac{4}{3}}} \lambda_{3}^{\mathrm{e}^{-\frac{2}{3}}}\right)+\frac{1}{3} \mu_{\text {neq }}\left(\lambda_{1}^{\mathrm{e}^{\frac{2}{3}}} \lambda_{3}^{\mathrm{e}^{-\frac{2}{3}}}-\lambda_{1}^{\mathrm{e}^{-\frac{4}{3}}} \lambda_{3}^{\mathrm{e}^{\frac{4}{3}}}\right) \\
& -\frac{1}{2 \mu_{0}} B_{3}^{2} \lambda_{1}^{-2} \lambda_{3}, \\
J \widetilde{\sigma}_{33} & =\frac{1}{2} \kappa_{\mathrm{eq}}\left(\lambda_{1}^{\frac{8}{3}} \lambda_{3}^{\frac{4}{3}}-\lambda_{1}^{-\frac{4}{3}} \lambda_{3}^{-\frac{2}{3}}\right)+\frac{2}{3} \mu_{\mathrm{eq}}\left(\lambda_{1}^{-\frac{4}{3}} \lambda_{3}^{\frac{4}{3}}-\lambda_{1}^{\frac{2}{3}} \lambda_{3}^{-\frac{2}{3}}\right) \\
& +\frac{1}{2} \kappa_{\mathrm{neq}}\left(\lambda_{1}^{\mathrm{e}^{\frac{8}{3}}} \lambda_{3}^{\mathrm{e}^{\frac{4}{3}}}-\lambda_{1}^{\mathrm{e}^{-\frac{4}{3}}} \lambda_{3}^{\mathrm{e}^{-\frac{2}{3}}}\right)+\frac{2}{3} \mu_{\mathrm{neq}}\left(\lambda_{1}^{\mathrm{e}^{-\frac{4}{3}}} \lambda_{3}^{\mathrm{e}^{\frac{4}{3}}}-\lambda_{1}^{\mathrm{e}^{\frac{2}{3}}} \lambda_{3}^{\mathrm{e}^{-\frac{2}{3}}}\right) \\
& +\frac{1}{2 \mu_{0}} B_{3}^{2} \lambda_{1}^{-2} \lambda_{3}+2 c_{2} B_{3}^{2} \lambda_{3}^{2}+4 c_{3} B_{3}^{2} \lambda_{3}^{4} \\
& +2 c_{5} B_{3}^{2} \lambda_{1}^{\mathrm{e}^{4}} \lambda_{3}^{\mathrm{e}^{2}} \lambda_{1}^{-4}+4 c_{6} B_{3}^{2} \lambda_{1}^{\mathrm{e}^{4}} \lambda_{3}^{\mathrm{e}^{4}} \lambda_{1}^{-4},
\end{aligned}
$$

with identical transverse stresses $\widetilde{\sigma}_{22}=\widetilde{\sigma}_{11}$, and where $\lambda_{1}^{\mathrm{e}}=\lambda_{1} \lambda_{1}^{\mathrm{v}^{-1}} \equiv \lambda_{2}^{\mathrm{e}}$ and $\lambda_{3}^{\mathrm{e}}=\lambda_{3} \lambda_{3}^{\mathrm{v}^{-1}}$ are the elastically relaxing stretches. 
Likewise, for the viscous deformation, the evolution equation (43) reduces in components to

$$
\begin{aligned}
\lambda_{1}^{\mathrm{e}^{4}} \frac{\cdot}{\log \lambda_{1}^{\mathrm{v}}} & =\frac{1}{2} \frac{\kappa_{\text {neq }}}{\eta}\left(\lambda_{1}^{\mathrm{e}^{\frac{8}{3}}} \lambda_{3}^{\mathrm{e}^{\frac{4}{3}}}-\lambda_{1}^{\mathrm{e}^{-\frac{4}{3}}} \lambda_{3}^{\mathrm{e}^{-\frac{2}{3}}}\right)+\frac{1}{3} \frac{\mu_{\text {neq }}}{\eta}\left(\lambda_{1}^{\mathrm{e}^{\frac{2}{3}}} \lambda_{3}^{\mathrm{e}^{-\frac{2}{3}}}-\lambda_{1}^{\mathrm{e}^{-\frac{4}{3}}} \lambda_{3}^{\mathrm{e}^{\frac{4}{3}}}\right) \\
& +2 \frac{c_{4}}{\eta} B_{3}^{2} \lambda_{1}^{\mathrm{e}^{4}} \lambda_{1}^{-4}+2 \frac{c_{5}}{\eta} B_{3}^{2} \lambda_{1}^{\mathrm{e}^{4}} \lambda_{3}^{\mathrm{e}^{2}} \lambda_{1}^{-4}+2 \frac{c_{6}}{\eta} B_{3}^{2} \lambda_{1}^{\mathrm{e}^{4}} \lambda_{3}^{\mathrm{e}^{4}} \lambda_{1}^{-4} \\
\lambda_{3}^{\mathrm{e}^{4}} \frac{\cdot}{\log \lambda_{3}^{\mathrm{v}}} & =\frac{1}{2} \frac{\kappa_{\text {neq }}}{\eta}\left(\lambda_{1}^{\mathrm{e}^{\frac{8}{3}}} \lambda_{3}^{\mathrm{e}^{\frac{4}{3}}}-\lambda_{1}^{\mathrm{e}^{-\frac{4}{3}}} \lambda_{3}^{\mathrm{e}^{-\frac{2}{3}}}\right)+\frac{2}{3} \frac{\mu_{\mathrm{neq}}}{\eta}\left(\lambda_{1}^{\mathrm{e}^{-\frac{4}{3}}} \lambda_{3}^{\mathrm{e}^{\frac{4}{3}}}-\lambda_{1}^{\mathrm{e}^{\frac{2}{3}}} \lambda_{3}^{\mathrm{e}^{-\frac{2}{3}}}\right) \\
& +2 \frac{c_{5}}{\eta} B_{3}^{2} \lambda_{1}^{\mathrm{e}^{4}} \lambda_{3}^{\mathrm{e}^{2}} \lambda_{1}^{-4}+4 \frac{c_{6}}{\eta} B_{3}^{2} \lambda_{1}^{\mathrm{e}^{4}} \lambda_{3}^{\mathrm{e}^{4}} \lambda_{1}^{-4}
\end{aligned}
$$

For the following analyses, we introduce the ratio $\beta=\kappa_{\text {neq }} / \mu_{\text {neq }}$ between the non-equilibrium bulk and shear moduli. Herein, the particular case of quasi-incompressibility in the viscous part of the response can be obtained with a relatively high value of $\beta$.

Remark 2. The (strong) hypothesis of homogeneous deformation is set here only for demonstrative purposes to focus on the intrinsic material response. At this stage, the applied magnetic induction is assumed given and the study reduces to that of a material point. Of course, in more realistic applications, the surrounding space together with the continuity conditions, i.e. (3) and $(9)_{2}$, must be accounted for in a more complete structural analysis, see for example ${ }^{34}$.

\subsection{Magnetic induction with no deformation}

In this first example, we consider that the above sample is held fixed with $\lambda_{1}=\lambda_{2}=\lambda_{3}=1$ during the whole loading history and we study the effect of an applied induction $B_{3}(t)$ on the purely viscoelastic part of the response, see the sketch of Fig. 2. To simplify the mathematical expressions, we introduce the following dimensionless magnetization parameters and variables:

$$
\alpha_{i}=\left.c_{i} \mu_{0}\right|_{i=1, \ldots, 6}, \quad \bar{B}=\frac{B_{3}}{\sqrt{\mu_{0} \mu_{\text {neq }}}}, \quad \bar{H}=H_{3} \sqrt{\frac{\mu_{0}}{\mu_{\text {neq }}}},
$$

where $\mathrm{H}_{3}$ is the $\vec{e}_{3}$-component of the corresponding magnetic field. From (47), one can observe that the viscoelastic stretches evolve with the characteristic time $\eta / \mu_{\text {neq }}$. This latter is herein used to normalize the time $t$ by introducing the dimensionless time $\tilde{t}=t \mu_{\text {neq }} / \eta$. With the constraints $\lambda_{1}=1$ and $\lambda_{3}=1$, 
the system (47) becomes

$$
\begin{aligned}
\lambda_{1}^{\mathrm{v}^{-4}} \frac{d}{d \tilde{t}}\left(\log \lambda_{1}^{\mathrm{v}}\right) & =\frac{\beta}{2}\left(\lambda_{1}^{\mathrm{v}^{-\frac{8}{3}}} \lambda_{3}^{\mathrm{v}^{-\frac{4}{3}}}-\lambda_{1}^{\mathrm{v}^{\frac{4}{3}}} \lambda_{3}^{\mathrm{v}^{\frac{2}{3}}}\right)+\frac{1}{3}\left(\lambda_{1}^{\mathrm{v}^{-\frac{2}{3}}} \lambda_{3}^{\mathrm{v}^{\frac{2}{3}}}-\lambda_{1}^{\mathrm{v}^{\frac{4}{3}}} \lambda_{3}^{\mathrm{v}^{-\frac{4}{3}}}\right) \\
& +2 \alpha_{4} \bar{B}^{2} \lambda_{1}^{\mathrm{v}^{-4}}+2 \alpha_{5} \bar{B}^{2} \lambda_{1}^{\mathrm{v}^{-4}} \lambda_{3}^{\mathrm{v}^{-2}}+2 \alpha_{6} \bar{B}^{2} \lambda_{1}^{\mathrm{v}^{-4}} \lambda_{3}^{\mathrm{v}^{-4}} \\
\lambda_{3}^{\mathrm{v}^{-4}} \frac{d}{d \tilde{t}}\left(\log \lambda_{3}^{\mathrm{v}}\right) & =\frac{\beta}{2}\left(\lambda_{1}^{\mathrm{v}^{-\frac{8}{3}}} \lambda_{3}^{\mathrm{v}^{-\frac{4}{3}}}-\lambda_{1}^{\mathrm{v}^{\frac{4}{3}}} \lambda_{3}^{\mathrm{v}}\right)+\frac{2}{3}\left(\lambda_{1}^{\mathrm{v}^{\frac{4}{3}}} \lambda_{3}^{\mathrm{v}^{-\frac{4}{3}}}-\lambda_{1}^{\mathrm{v}^{-\frac{2}{3}}} \lambda_{3}^{\mathrm{v}^{\frac{2}{3}}}\right) \\
& +2 \alpha_{5} \bar{B}^{2} \lambda_{1}^{\mathrm{v}^{-4}} \lambda_{3}^{\mathrm{v}^{-2}}+4 \alpha_{6} \bar{B}^{2} \lambda_{1}^{\mathrm{v}^{-4}} \lambda_{3}^{\mathrm{v}^{-4}},
\end{aligned}
$$

which is solved for $\lambda_{1}^{\mathrm{v}}(\tilde{t})$ and $\lambda_{3}^{\mathrm{v}}(\tilde{t})$, and by mere function evaluation using these latter into (46), one can also deduce the corresponding dimensionless total stress components $\widetilde{\sigma}_{11} / \mu_{\text {neq }}$ and $\widetilde{\sigma}_{33} / \mu_{\text {neq }}$.

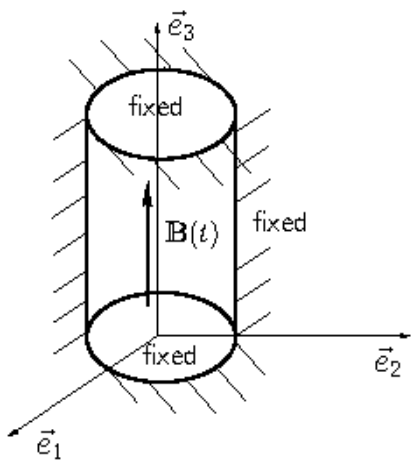

Figure 2. Fixed cylindrical sample and magnetic induction loading.

Some representative solutions to the differential-algebraic system (49) are plotted in Fig. 3 for the case where the dimensionless induction is applied quickly at a relatively high rate $d \bar{B} / d \tilde{t}=100$ until it reaches the value $\bar{B}=1$, and then is left fixed at this value. At dimensionless time $\tilde{t}=0$, the sample is fully relaxed with the initial conditions being $\lambda_{i}=\lambda_{i}^{\mathrm{v}}=1, i=1,2,3$. Here as the system (49) is independent on the equilibrium magnetization parameters, we choose to set them to zero as $\alpha_{1}=\alpha_{2}=\alpha_{3}=0$, so that the dimensionless magnetic field $(48)_{3}$ is linked to the dimensionless induction by the relation

$$
\bar{H}=\left[\frac{2 \alpha_{4}}{\lambda_{1}^{\mathrm{v}^{4}}}+\frac{2 \alpha_{5}}{\lambda_{1}^{\mathrm{v}^{4}} \lambda_{3}^{\mathrm{v}^{2}}}+\frac{2 \alpha_{6}}{\lambda_{1}^{\mathrm{v}^{4}} \lambda_{3}^{\mathrm{v}^{4}}}+1\right] \bar{B} .
$$

We study the influence of the non-equilibrium magnetization parameters $\alpha_{4}, \alpha_{5}$ and $\alpha_{6}$ (or equivalently of $c_{4}, c_{5}$ and $c_{6}$ ) by choosing to activate only one of them each time. Each case is computed with three different ranges of compressibility in the mechanical viscoelastic part through the ratio $\beta$. We choose the 

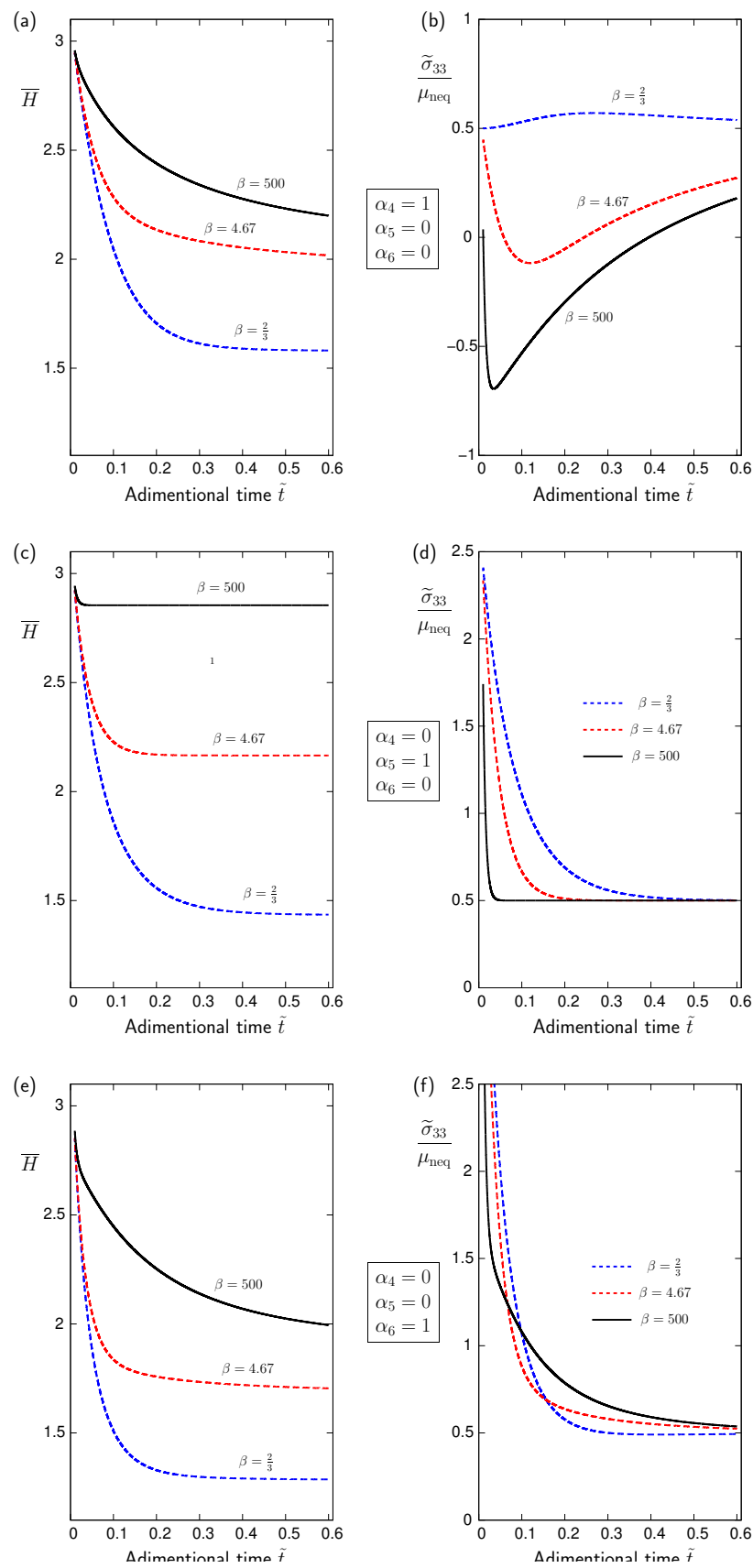

Figure 3. (a) (c) (e) Dimensionless magnetic field evolution $\bar{H}(\tilde{t})$ and corresponding (b) (d) (f) dimensionless total stress $\widetilde{\sigma}_{33}(\tilde{t}) / \mu_{\text {neq. }}$. In each case, only one non equilibrium magnetization parameter is activated: $\alpha_{4}$ for (a-b), $\alpha_{5}$ for (c-d), and $\alpha_{6}$ for (e-f). Three different ratios $\beta=2 / 3,4.67$, and 500 are used for each calculation. 
values $\beta=2 / 3$, that corresponds to a zero Poisson's ratio in the limiting case of linearized kinematics, $\beta=4.67$ that corresponds to an approximate 0.4 Poisson's ratio, also in the limiting case of linearized kinematic, and $\beta=500$ that corresponds to a viscoelastic quasi-incompressibility within the whole strain range.

One can see from Figs. 3(a)(c)(e) that, in all cases, the viscoelastic compressibility strongly influences the decay to equilibrium of the magnetic field. For the augmented Cauchy stress, Fig. 3(b) shows an unexpected evolution with no decay. This is explained by the fact that the stress is independent of the parameter $\alpha_{4}$, or $c_{4}$, see Eq. $(46)_{2}$. On the other side, for the cases where $\alpha_{5}$ and/or $\alpha_{6}$ are activated, it is seen from Figs. 3(d)(f) that the stress decays to an equilibrium value as expected. This decay is particularly sharp for $\alpha_{5}=1$ in the limiting case of incompressibility, i.e. the curve with $\beta=500$ in Fig. 3(d), and likewise for the corresponding magnetic field's decay in Fig. 3(c).

\subsection{Responses to cyclic loads}

In this second example, the dynamic responses of the above cylindrical sample are studied. The sample is this time unconstrained with free lateral and axial stretches and we still stipulate the homogeneous assumption for the purpose of demonstration and qualitative prediction, see Fig. 4. Initially, the sample is fully relaxed at $t=0$, with the initial conditions $\lambda_{i}=\lambda_{i}^{\mathrm{v}}=1, i=1,2,3$.

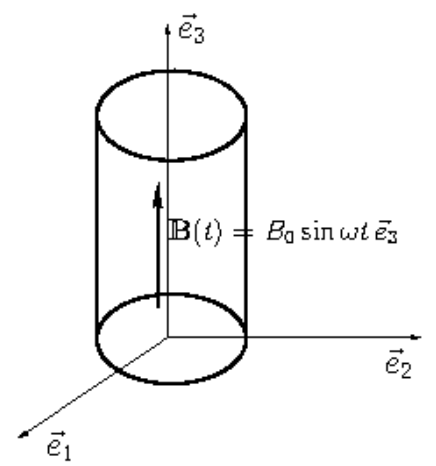

Figure 4. Free cylindrical sample and sinusoidal magnetic induction loading.

In this case four equations constitute the differential-algebraic system to be solved for the stretches $\lambda_{1}, \lambda_{1}^{\mathrm{v}}, \lambda_{3}$ and $\lambda_{3}^{\mathrm{v}}$ : the two issued from the mechanical balance, $\widetilde{\sigma}_{11}=0$ and $\widetilde{\sigma}_{33}=0$ in the absence of mechanical body forces, and the two evolution equations (47). The expressions of the formers are given in (46). 
Here again, we simplify the mathematical expressions with an adimensionalization procedure as follows. While conserving the definitions $(48)_{1}$ for the dimensionless magnetization parameters $\alpha_{i}$, $i=1 \ldots 6$, the dimensionless variables are this time defined as

$$
\bar{B}=\frac{B_{3}}{\sqrt{\mu_{0} \mu}}, \quad \text { and } \bar{H}=H_{3} \sqrt{\frac{\mu_{0}}{\mu}},
$$

where $\mu=\mu_{\mathrm{eq}}+\mu_{\mathrm{neq}}$ is the instantaneous shear modulus of the material and, to simplify further, we also consider the same ratio between the couples of bulk and shear moduli of the equilibrium and nonequilibrium parts, i.e.

$$
\beta=\frac{\kappa_{\text {neq }}}{\mu_{\text {neq }}} \equiv \frac{\kappa_{\text {eq }}}{\mu_{\text {eq }}}
$$

Now defining $\zeta=\mu_{\mathrm{eq}} / \mu \in[0,1]$ as the ratio between the equilibrium and instantaneous shear moduli, we deduce the following relations for the material parameters that will also be used when adimensionalizing the equilibrium equations $\widetilde{\sigma}_{11}=0$ and $\widetilde{\sigma}_{33}=0$ by $\mu$ :

$$
\frac{\mu_{\mathrm{neq}}}{\mu}=1-\zeta, \quad \frac{\kappa_{\mathrm{eq}}}{\mu}=\beta \zeta, \quad \text { and } \frac{\kappa_{\mathrm{neq}}}{\mu}=\beta(1-\zeta)
$$

Last, as for the example in Section 4.1, the evolution equations (47) are here again normalized with the adimensional time $\tilde{t}=t \mu_{\text {neq }} / \eta$.

We write the applied dimensionless magnetic induction into a sinusoidal function $\bar{B}=\bar{B}_{0}$ sin $\omega \tilde{t}$, with $\omega$ and $\bar{B}_{0}$ being the dimensionless frequency and amplitude, respectively. A set of representative results are presented in Fig. 5. In all the solutions plotted, the amplitude is set to be $\bar{B}_{0}=2$, we consider a quasi-incompressible material with $\beta=500$, and the material parameter $\zeta$ is taken to be 0.5 so that the equilibrium and non-equilibrium contributions are comparable. Hence, we study the influence of the magnetization parameters $\alpha_{i}, i=1 \ldots 6$, together with the driving induction frequency $\omega$.

For illustrative purposes, we choose here again to activate only one non-equilibrium magnetization parameter each time; $\alpha_{5}$ in Figs. 5 (a-b), $\alpha_{6}$ in Figs. 5 (c-d), and a combination of this latter with the activation of an equilibrium parameter, for instance, here with $\alpha_{3}$ in Figs. 5 (e-f). In the absence of further investigations, we prefer to not consider the non-equilibrium parameter $\alpha_{4}$ due to its unexpected influence seen in the first example, Fig. 3 (b). For the sake of comparison, two frequencies are used; a moderate one with $\omega=1$, and a relatively high one with $\omega=10$.

The first observation is that, for the moderate frequency, the stretches stabilize in 1-2 cycles, while for the relatively high frequency, the stabilization takes place after 10-12 cycles. In each case, the amplitude of the viscoelastic stretch $\lambda_{3}^{\mathrm{v}}$ is markedly smaller than that of $\lambda_{3}$. Moreover, for each set of parameters, the amplitude of $\lambda_{3}^{\mathrm{v}}$ is markedly smaller for the high frequency than for the moderate one, but the mean 

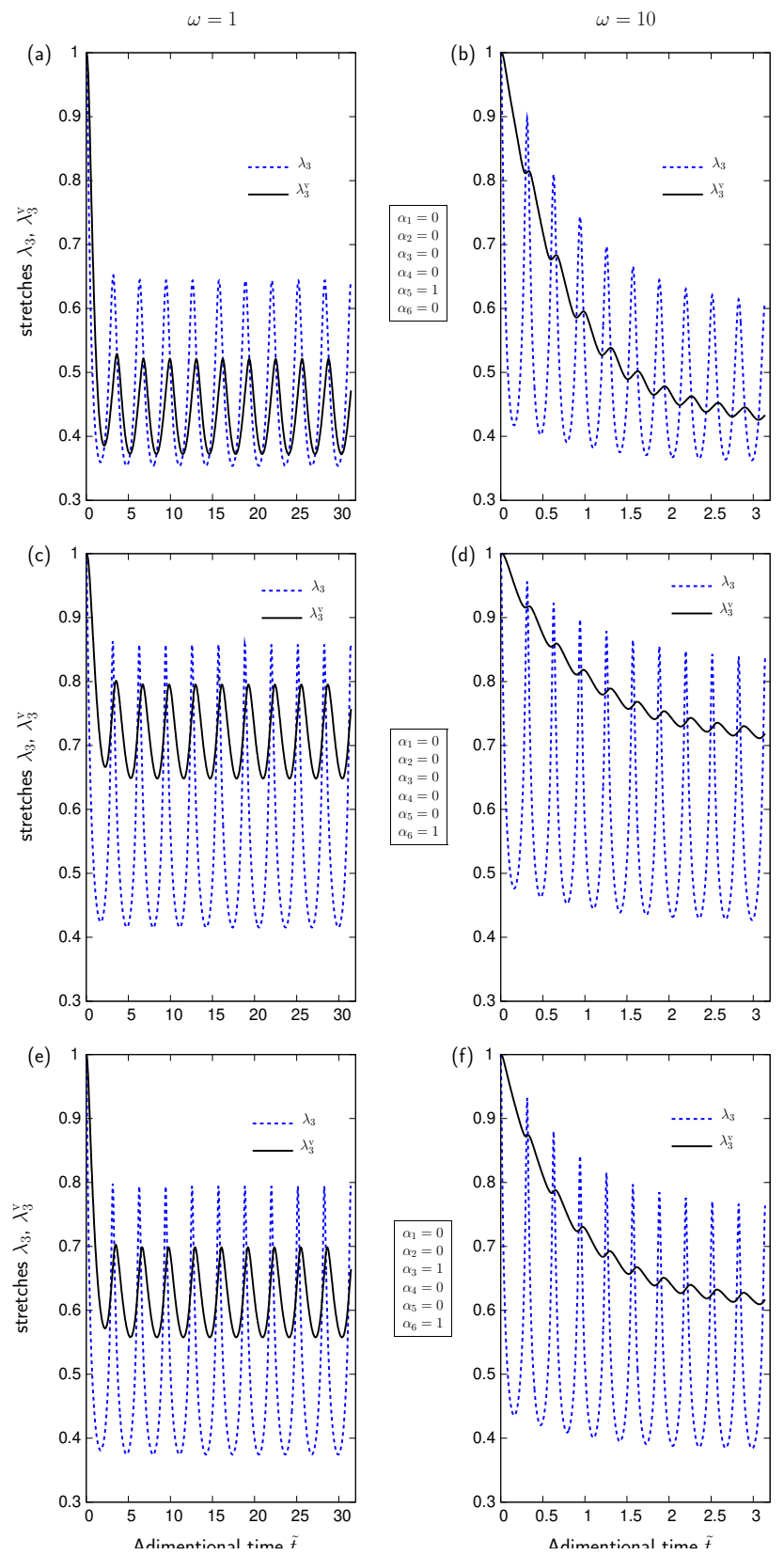

Figure 5. Total and viscoelastic stretches $\lambda_{3}(\tilde{t})$ and $\lambda_{3}^{\mathrm{v}}(\tilde{t})$ in response to applied sinusoidal magnetic induction with frequency $\omega=1$ in (a) (c) (e), and $\omega=10$ in (b) (d) (f). Each time with the activation of different magnetization parameters, as indicated. 
value at stabilization seems to be the same. As a last remark, it can be seen from Figs. 5 (b) and (d) that, with $\alpha_{5}$, the mean stretching $\lambda_{3}^{\mathrm{v}}$ is higher than the mean total one $\lambda_{3}$, while with $\alpha_{6}$, the mean $\lambda_{3}^{\mathrm{v}}$ is lower that $\lambda_{3}$. We remember that sample experiences contraction in the $\vec{e}_{3}$-direction under the applied magnetic load, hence the stretches $\lambda_{3}$ and $\lambda_{3}^{\mathrm{v}}$ are lower than 1 .

\section{Conclusion and perspectives}

In this paper, we have presented a general theory to model the nonlinear magneto-viscoelasticity in the finite strain range. Within the framework of continuum thermodynamics, the nowadays well know fields related to the magnetic coupling have been embedded in a sound way for a concise characterization of the whole set of constitutive equations.

The viscoelastic kinematics adopted herein is based on the multiplicative decomposition of the deformation gradient into an elastically relaxing part and a viscous part. The formulation developed in this paper is based on the magnetic induction vector as main independent variable for the magnetic part of the coupled problem and, to make matters as concrete as possible, a model problem has been proposed for demonstrative purposes so as to conduct preliminary parametric studies to qualitatively show some basic phenomena revealed by the formulation.

We believe that further work has to be accomplished to optimize the present theory toward more realistic modelling of smart magneto-active materials. Experimental investigations will certainly give better knowledge of the material properties. Among others, the magnetization parameters must be quantified. Experimental tests could for example show which ones are most relevant for the modelling or, at least, whether or not the non-equilibrium ones are pertinent. On the other hand, as most of the MAPs are manufactured as anisotropic composites, the present modelling framework must be extended by taking into account the directional dependencies of the magneto-viscoelastic responses.

\section{References}

1. Ginder JM, Clark SM, Schlotter WF et al. Magnetostrictive phenomena in magnetorheological elastomers. International Journal of Modern Physics B 2002; 16: 2412-2418.

2. Jolly MR, Carlson JD and Muñoz BC. A model of the behavior of magnetorheological materials. Smart Materials and Structures 1996; 5: 607-614.

3. Varga Z, Filipcsei G and Zrínyi M. Magnetic field sensitive functional elastomers with tunable elastic modulus. Polymer 2006; 47: 227-233.

4. Zrínyi M, Barsi L and Büki A. Deformation of ferrogels induced by nonuniform magnetic fields. Journal of Chemical Physics 1996; 104(21): 8750-8756. 
5. Ren Z, Hu W, Dong X et al. Multi-functional soft-bodies jellyfish-like swimming. Nature Communications 2019; 10: Article number: 2703.

6. $\mathrm{Hu}$ W, Zhan G, Mastrangeli M et al. Small-scale soft-bodied robot with multimodal locomotion. Nature 2018; 554: 81-85.

7. Lu H, Zhang M, Yang $\mathrm{Y}$ et al. A bioinspired multilegged soft millirobot that functions in both dry and wet conditions. Nature Communications 2018; 9: Article number: 3944.

8. Brigadnov IA and Dorfmann A. Mathematical modeling of magneto-sensitive elastomers. International Journal of Solids and Structures 2003; 40: 4659-4674.

9. Bustamante R, Dorfmann A and Ogden RW. Universal relations in isotropic nonlinear magnetoelasticity. The Quarterly Journal of Mechanics and Applied Mathematics 2006; 59: 435-450.

10. Dorfmann A and Ogden RW. Magnetoelastic modelling of elastomers. European Journal of Mechanics A/Solids 2003; 22: 497-507.

11. Dorfmann A and Ogden RW. Nonlinear magnetoelastic deformations. Quarterly Journal of Mechanics and Applied Mathematics 2004; 57: 599-622.

12. Dorfmann A and Ogden RW. Nonlinear magnetoelastic deformations of elastomers. Acta Mechanica 2004; 167(1-2): 13-28.

13. Ericksen JL. A modified theory of magnetic effects in elastic materials. Mathematics and Mechanics of Solids 2006; 11: 23-47.

14. Steigmann DJ. Equilibrium theory for magnetic elastomers and magnetoelastic membranes. International Journal of Non-Linear Mechanics 2004; 39: 1193-1216.

15. Vu DK and Steinmann P. Nonlinear electro- and magneto-elastostatics: Material and spatial settings. International Journal of Solids ansd Structures 2007; 44: 7891-7901.

16. Nedjar B. A theory of finite strain magneto-poromechanics. Journal of the Mechanics and Physics of Solids 2015; 84: 293-312.

17. Ogden RW. Magnetostatics: from basic principles to nonlinear interactions in deformable media. In Ogden $\mathrm{R}$ and Steigmann D (eds.) Mechanics and Elastodynamics of Magneto- and Electro-elastic Materials, CISM Courses and Lectures No. 527. Spinger, Wien, NewYork, pp. 107-152.

18. Brown WF. Magnetoelastic Interactions. Berlin: Springer, 1966.

19. Jackson JD. Classical Electrodynamics, 3rd edition. New York: John Wiley \& Sons, 1999.

20. Pao YH. Electromechanic forces in deformable continua. In Nemat-Nasser S (ed.) Mechanics Today, vol. 4. Oxford University Press, pp. 209-305.

21. Amin AFMS, Lion A, Sekita S et al. Nonlinear dependence of viscosity in modeling the rate-dependent response of natural and high damping rubbers in compression and shear: Experimental identification and numerical verification. International Journal of Plasticity 2006; 22(9): 1610-1657. 
22. Liao Z, Yao X, Zhang L et al. Temperature and strain dependent large tensile deformation and tensile failure behavior of transparent polyurethane at intermediate strain rates. International Journal of Impact Engineering 2019; 129: 152-167.

23. Pelteret JP, Walter B and Steinmann P. Application of metaheuristic algorithms to the identification of nonlinear magneto-viscoelastic constitutive parameters. Journal of Magnetism and Magnetic Materials 2018; 464: 116131.

24. Yunus NA, Mazlan SA, Ubaidillah U et al. Thermal stability and rheological properties of epoxidized natural rubber-based magnetorheological elastomer. International Journal of Molecular Sciences 2019; 20(3): Article number: 746.

25. Govindjee $\mathrm{S}$ and Reese S. A presentation and comparison of two large deformation viscoelastic models. Journal of Engineering Materials and Technology 1997; 119: 251-255.

26. Hong W. Modeling viscoelastic dielectrics. Journal of the Mechanics and Physics of Solids 2011; 59: 637-650.

27. Nedjar B. Framework for finite strain viscoelastic-plasticity based on multiplicative decompositions: Part I: continuum formulations. Computer Methods in Applied Mechanics and Engineering 2002; 191: 1541-1562.

28. Nedjar B. Framework for finite strain viscoelastic-plasticity based on multiplicative decompositions: Part II: computational aspects. Computer Methods in Applied Mechanics and Engineering 2002; 191: 1563-1593.

29. Reese S and Govindjee S. A theory of finite viscoelasticity and numerical aspects. International Journal of Solids and Structures 1998; 35(26-27): 3455-3482.

30. Sidoroff F. Un modèle viscoélastique nonlinéaire avec configuration intermédiaire. Journal de Mécanique 1974; 13: $679-713$.

31. Saxena P, Hossain M and Steinmann P. A theory of finite deformation magneto-viscoelasticity. International Journal of Solids and Structures 2013; 50: 3886-3897.

32. Han Y, Hong W and Faidley L. Coupled magnetic field and viscoelasticity of ferrogels. International Journal of Applied Mechanics 2011; 3(2): 259-278.

33. Nedjar B. A finite strain modeling of electro-viscoelastic materials. International Journal of Solids and Structures 2016; 97-98: 312-321.

34. Nedjar B. A couple BEM-FEM method for finite strain magneto-elastic boundary-value problems. Computational Mechanics 2017; 59(5): 795-807.

35. Coleman BD and Gurtin ME. Thermodynamics with internal variables. Journal of Chemics and Physics 1967; 47: 597-613.

36. Bustamante R, Dorfmann A and Ogden RW. On variational formulations in nonlinear magnetoelastostatics. Mathematics and Mechanics of Solids 2008; 13: 725-745.

37. Lubliner J. A model of rubber viscoelasticity. Mechanics Research Communications 1985; 12: 93-99.

38. Holzapfel GA. Nonlinear Solid Mechanics. A Continuum Appraoch for Engineering. Chichester, West Sussex, UK: John Wiley and Sons, Ltd, 2000.

Prepared using sagej.cls 
39. Marsden JE and Hughes TJR. Mathematical Foundations of Elasticity. Englewood-Cliffs, NJ: Prentice-Hall, 1983.

40. Boyce MC, Weber GC and Parks DM. On the kinematics of finite strain plasticity. Journal of the Mechanics and Physics of Solids 1989; 37(5): 641-665.

41. Nedjar B. An anisotropic viscoelastic fibre-matrix model at finite strains: Continuum formulation and computational aspects. Computer Methods in Applied Mechanics and Engineering 2007; 196(9-12): 17451756.

42. Spencer AJM. Constitutive theory for strongly anisotropic solids. In Spencer AJM (ed.) Continuum Theory of the Mechanics of Fibre-reinforced Composites, CISM Cources and Lectures No. 282. Springer, Wien.

43. Zhou J, Jiang L and Khayat RE. A micro-macro constitutive model for finite-deformation viscoelasticity of elastomers with nonlinear viscosity. Journal of the Mechanics and Physics of Solids 2018; 110: 137-154.

44. Nedjar B. On constitutive models of finite elasticity with possible zero apparent Poisson's ratio. International Journal of Solids and Structures 2016; 91: 72-77.

45. Nedjar B, Baaser H, Martin RJ et al. A finite element implementation of the isotropic exponentiated henckylogarithmic model and simulation of the eversion of elastic tubes. Computational Mechanics 2018; 62(4): $635-654$. 